\title{
Orofacial Cysts at Komfo Anokye Teaching Hospital, Ghana
}

\author{
Alexander Acheampong Oti $^{{ }^{*}}$, Peter Donkor ${ }^{2}$, Osei Owusu-Afriyie ${ }^{1}$ \\ ${ }^{1}$ Komfo Anokye Teaching Hospital, Kumasi, Ghana \\ ${ }^{2}$ Kwame Nkrumah University of Science and Technology, Kumasi, Ghana \\ Email: *aotiacheampong@yahoo.com
}

Received November 11, 2012; revised December 13, 2012; accepted December 22, 2012

\begin{abstract}
Background: Information about orofacial cysts from African populations is scarce and there are only a few studies available regarding the prevalence of these lesions in the West African sub-region. The purpose of the present study is to determine the distribution and prevalence of all histologically diagnosed orofacial cysts in Kumasi, Ghana. Aim: To determine prevalence, sex, age and anatomic distribution of orofacial cyst seen at the oral and maxillofacial unit in Komfo Anokye Teaching hospital (KATH). Method: This is a retrospective study, which examined histologically diagnosed lesions including orofacial cysts. The study duration was from 1999 to 2010 September inclusive. Results: There were 37 odontogenic cysts constuting $6.5 \%$, of all orofacial lesions. There were 18 non-odontogenic cysts i.e. $3.1 \%$ of all lesions diagnosed during the study period. The odontogenic cysts comprised 19 (51.4\%) developmental cysts and 18 (48.6\%) inflammatory cysts. Male-to-female ratio for the orofacial cysts was 1:1 and the mean age was 36.7 years. Conclusion: There is low prevalence of the odontogenic cysts, which is consistent with findings from other African studies. Although radicular cysts accounted for the majority of orofacial cysts in this study, the prevalence of radicular cysts is low compared to reports from developed countries.
\end{abstract}

Keywords: Orofacial; Cyst; Radicular; Odontogenic

\section{Introduction}

Orofacial cysts are broadly divided into odontogenic and non-odontogenic types. The odontogenic cysts is an osseous-destructive lesion, which more commonly affects the jaws [1]. It is considered that proliferation and cystic degeneration of odontogenic epithelium leads to the development of odontogenic cysts [1,2].

There are different types of odontogenic cysts: Odontogenic keratocyst occurs in patients ranging in age from infancy to old age [3]. By definition, a dentigerous cyst occurs in association with an unerupted tooth, most commonly mandibular third molars. Trauma to dental structures and infection can cause inflammatory reaction at the periapical region of the tooth, leading to the formation of the inflammatory type of odontogenic cyst.

Epithelial remnant proliferations of non-odontogenic origin are responsible for the formation of non-odontogenic cysts. Aneurysmal bone cysts are relatively rare osteolytic lesions. They are mainly found within the long bones, and only $2 \%$ occur in the jaws [4]. They occur in patients of all ages, with, a slightly higher prevalence in the first decades [5-7]. The mandible is more prone to

*Corresponding author. being affected [8]. The purpose of the present study is to determine the demographic and anatomic distribution of odontogenic and non-odontogenic cysts affecting the orofacial region over a 11-year period at the in Komfo Anokye Teaching Hospital in Kumasi-Ghana and it's environ which serves a population of about four million.

\section{Methodology}

This is a retrospective study of oro-facial cysts seen at the Maxillofacial Unit, Komfo Anokye Teaching Hospital, Ghana from 1999 to September 2010. Information from histopathology reports including age, sex, histological diagnosis and anatomic location was extracted on to data collection forms.

\section{Study Site}

The study site is the oral and maxillofacial surgery unit of the Komfo Anokye Teaching Hospital, Ghana.

\section{Statistical Analysis}

Data from the reports were entered into a spreadsheet and analyzed using the Statistical Package for social science, version 16.0 (SPSS, IL). Frequencies and means were 
determined using descriptive analysis.

\section{Results}

Orofacial cysts constituted 9.6\% out of the 567 the histopathologically diagnosed oro-facial lesions. Male-female distribution for all orofacial cyst was approximately in the ratio of $1: 1$ and the mean age was 36.7 years. The orofacial cysts comprised 37 (67.3\%) odontogenic cysts, and 18 (32.7\%) non-odontogenic cysts. The odontogenic cysts included 19 (51.4\%) developmental cysts and 18 (48.6\%) inflammatory cysts. Of the total 19 cases of the developmental odontogenic cysts, 16 (84.2\%) occurred in the mandible. For odontogenic keratocyst the male to female ratio was 1:2 and the peak age range was 21 to 30 years. Dentigerous cysts had male-female ratio of 2.3:1 and peak age range of 11 to 20 years accounting for $40 \%$.

The commonest non-odontogenic cyst was nasolabial cyst, representing $61 \%$ of all non-odontogenic cysts. The nasolabial and nasopalatine cyst had almost equal sex distribution and were located exclusively in the maxilla. The peak age range for the nasolabial cysts was in the 31 50 years while that for nasopalatine cysts was 21 - 30 years. All the aneurysmal bone cysts were located in the mandible with male-female ratio of 2:1 and peak age range of 21 - 30 years.

\section{Discussion}

There is very little published information on the prevalence of oro-facial cysts in Ghana. This study will therefore contribute to the relevant literature on cysts. The mean age for all the oro-facial cysts was 36.5 years, and is comparable to finding from a study from Brazil [9]. A recent study in Kuwait [10] showed that oro-facial cysts made up $46.3 \%$ of all biopsies of jaw lesions as compared with $9.6 \%$ from this study. The prevalence was $13.2 \%$ in Zimbabwe [11] and 15.2\% in Brazil [9]. In the Brazilian study [9], (96.7\%) of the cysts were of odontogenic origin and 3.3\% were non-odontogenic. By comparison, odontogenic cysts made up $67.3 \%$ of cysts and odontogenic cysts $32.7 \%$. Studies from Canada [1] and the United Kingdom [12] showed prevalence rates of $17.2 \%$ and $12.8 \%$ respectively for odontogenic cysts. This low prevalence of odontogenic cysts could be explained by the fact that Ghana which is a developing country has a lower incidence of caries compared to the more developed countries and also radicular cyst which is an inflammatory cyst accounted for the majority of odontogenic cysts seen in most of the developed countries like Ulm-Germany [13] (54\%) but only $29.7 \%$ in this study, which also hap-pens to be the commonest odontogenic cyst in this study. Also from the authors' observation in Ghana, most of periapical soft tissues sur- rounding extracted teeth are not taken for histologic diagnosis unless a neoplasm is suspected.

Thus a large number of the inflammatory odontogenic cysts especially radicular cysts are missed.

The non-odontogenic cysts in this study represented $3.1 \%$ of all histologically diagnosed oro-facial lesions, while they represented $1.01 \%$ of lesions in Canada [1].

Nasolabial and nasopalatine cysts were located only in the maxilla, which is consistent with a similar study in Zimbabwe [11]. However, more nasolabial cysts were seen in this study compared to the Zimbabwe study.

Although dentigerous cysts occur over a wide age range, they are most commonly seen in 10 to 30 year olds [2]. Ninety percent (90\%) were in the age range of 10 to 30 years with peak and mean age of 23.2 years which is slightly less than the 27.4 years observed in Kuwait [10]. In this study, dentigerous cysts represent $27.1 \%$ of odontogenic cysts with male to female ratio of $1: 1.5$, and is similar to the Brazil [9], Kuwait [10] and Zimbabwe [11] studies. Eighty percent (80\%) of the dentigerous cysts were located in the mandible as in other African studies [11,14].

In the Kuwait study more males than females were affected as in the present study. In a study in Nigeria [14], out of a total 20 oral cysts, 10 (50.0\%) were dentigerous cysts and showed similar gender and age distribution as the present study. The difference in prevalence between the two studies could be due to the small sample size in the Nigerian study.

\section{Odontogenic Keratocyst (OKC)}

In this study OKC represented $1.6 \%$ of the jaw cysts, a prevalence which is similar to $1.3 \%$ from Sicily [15] but different from $4.4 \%$ in Zimbabwe [11]. More females were affected with male-female ratio of 1:1.5. The peak age range was 21 to 30 years and mean age of 32.2 years. This was lower than 39.1 years in Sicily [15] and 34.1 years in Kuwait [10].

\section{Inflammatory Cyst}

Radicular cyst is the commonest of the odontogenic cysts, accounting for $30 \%$ of odontogenic cysts as in Zimbabwe [11] study but far lower than 61\% in Brazil [9], 54\% in Kuwait [10]. This is, however, higher than the $15 \%$ observed in a Nigerian study [14]. The differences in prevalence between the studies from Africa and other more developed countries could be due to the relative lack of histopathological services diagnosis laboratories in many hospitals in the region. Thus, lesions around extracted teeth are rarely submitted for histological analysis unless they are large enough to warrant suspicion of malignancy. As in other studies, most of the radicular cysts were located in the mandible (82\%). 


\section{Aneurysmal Bone Cysts}

Aneurysmal bone cysts formed $16 \%$ of all non-odontogenic cysts, with male-female ratio of 2:1 and were only seen in the mandible as in other studies where $88.2 \%$ were located in the mandible $[5,6]$. The peak age range in this present study of 11 - 30 years and peak age range of 21 - 30 years with mean age of 24.1 years is slightly higher than what was observed by Motamedi [4].

Some studies demonstrate slight female predilection [7] which is different from this current study where all those affected were male as was the case in a Zimbabwe study [11]. As far as location is concerned, some studies show that, the cyst affects the mandible more often with a predominant location in the posterior part and the mandibular ramus [4,11].

\section{Conclusion}

There was low prevalence of the odontogenic cysts in this part of Ghana, as in other African countries. Although radicular cysts accounted for the majority of orofacial cysts in this present study, the prevalence of radicular cysts was low compared to most developed countries. This could be due to underdiagnosis, though a larger study is required to clarify the true occurrence.

\section{REFERENCES}

[1] T. D. Daley, G. P. Wysocki and G. A. Pringle, "Relative Incidence of Odontogenic Tumors and Oral and Jaw Cysts in a Canadian Population," Oral Surgery, Oral Medicine, Oral Pathology, Vol. 77, No. 3, 1994, pp. 276-280. doi:10.1016/0030-4220(94)90299-2

[2] A. Mosqueda-Taylor, M. E. Irigoyen-Camacho, M. A. Diaz-Franco and M. A. Torres-Tejero, "Odontogenic Cysts. Analysis of 856 Cases,” Medicina Oral, Vol. 7, No. 2, 2002, pp. 89-96.

[3] J. G. Meara, K. K. Li, S. S. Shah and M. J. Cunningham, "Odontogenic Keratocysts in the Pediatric Population," Archives of Otolaryngology Head and Neck Surgery, Vol. 122, No. 7, 1996, pp. 725-728. doi:10.1001/archotol.1996.01890190021006

[4] M. H. K. Motamedi, F. Navi, P. S. Eshkevari, S. M. Jafari, M. G. Shams, M. Taheri, F. M. Abbas and P. Motahhari, "Variable Presentations of Aneurysmal Bone Cysts of the Jaws: 51 Cases Treated during a 30-Year Period,” Journal of Oral and Maxillofacial Surgery, Vol. 66, No. 10, 2008, pp. 2098-2103. doi:10.1016/j.joms.2008.05.364

[5] Z. J. Sun, H. L. Sun, R. L. Yang, R. A. Zwahlen and Y. F. Zhao, "Aneurysmal Bone Cysts of the Jaws," Interna- tional Journal of Surgical Pathology, Vol. 17, No. 4, 2009, pp. 311-322. doi:10.1177/1066896909332115

[6] Z.-J. Sun, Y.-F. Zhao, R.-L. Yang and R. A. Zwahlen, "Aneurysmal Bone Cysts of the Jaws: Analysis of 17 Cases,” Journal of Oral and Maxillofacial Surgery, Vol. 68, No. 9, 2010, pp. 2122-2128. doi:10.1016/j.joms.2009.07.111

[7] W. M. Mendenhall, R. A. Zlotecki, C. P. Gibbs, J. D. Reith, M. T. Scarborough and N. P. Mendenhall, "Aneurysmal Bone Cyst,” American Journal of Clinical Oncology, Vol. 29, No. 3, 2006, pp. 311-315. doi:10.1097/01.coc.0000204403.13451.52

[8] J. M. López-Arcas Calleja, J. L. Cebrián Carretero, J. G. Martín and M. Burgueño, “Aneurysmal Bone Cyst of the Mandible: Case Presentation and Review of the Literature,” Medicina Oral, Patología Oral y Cirugía Bucal, Vol. 12, No. 5, 2007, pp. E401-E403.

[9] S. M. Grossmann, V. C. Machado, G. M. Xavier, M. D. Moura, R. S. Gomez, M. C. F. Aguiar and R. A. Mesquita, "Demographic Profile of Odontogenic and Selected Nonodontogenic Cysts in a Brazilian Population,” Oral Surgery, Oral Medicine, Oral Pathology, Oral Radiology, Vol. 104, No. 6, 2007, pp. e35-e41. doi:10.1016/j.tripleo.2007.05.028

[10] M. A. Ali, "Biopsied Jaw Lesions in Kuwait: A Six-Year Retrospective Analysis,” Medical Principles and Practice, Vol. 20, No. 6, 2011, pp. 550-555. doi:10.1159/000330023

[11] M. M. Chidzonga, V. M. Lopez and A. L. P. Alvarez, "Orofacial Biopsies: A Survey of 1723 Cases Seen over a 10 Year Period,” Central African Journal of Medicine, Vol. 42, No. 4, 1996, pp. 102-108.

[12] A. V. Jones, G. T. Craig and C. D. Franklin, "Range and Demographics of Odontogenic Cysts Diagnosed in a UK Population over a 30-Year Period,” Journal of Oral Pathology \& Medicine, Vol. 35, No. 8, 2006, pp. 500-507. doi:10.1111/j.1600-0714.2006.00455.x

[13] J. F. Kreidler, E. J. Raubenheimer and W. F. van Heerden, "A Retrospective Analysis of 367 Cystic Lesions of the Jaw-The Ulm Experience,” Journal of Cranio-Maxillofacial Surgery, Vol. 21, No. 8, 1993, pp. 339-341. doi:10.1016/S1010-5182(05)80494-9

[14] C. Oji, "Statistical Observations on Jaw Cysts in Enugu, Nigeria, 1987-1996,” Odontostomatol Trop, Vol. 22, No. 85, 1999, pp. 33-36.

[15] S. Tortorici, E. Amodio, M. F. Massenti, M. L. Buzzanca, F. Burruano and F. Vitale, "Prevalence and Distribution of Odontogenic Cysts in Sicily: 1986-2005," Journal of Oral Science, Vol. 50, No. 1, 2008, pp. 15-18. doi:10.2334/josnusd.50.15 